REVIEW

\title{
Multidetector row computed tomography: imaging congenital coronary artery anomalies in adults
}

\author{
N E Manghat, G J Morgan-Hughes, A J Marshall, C A Roobottom
}

Heart 2005;91:1515-1522. doi: 10.1136/hrt.2005.065979

The quality of the imaging of the main coronary arteries and side branches provided by multidetector row computed tomography (MDCT) may have importance when assessing congenital coronary artery anomalies. This review discusses the rationale for using MDCT for this indication and examines the advantages and disadvantages of the technique. Examples of MDCT imaging of congenital coronary artery anomalies are presented. These images provide persuasive evidence to support clinical use of MDCT cardiac imaging in the context of suspected congenital coronary artery anomalies as a first line investigation.

See end of article for authors' affiliations

Correspondence to: Dr Nathan E Manghat, Department of Clinical' Radiology, Plymouth NHS Trust, Derriford, Plymouth PL6 8DH, UK; docnatman@msn.com

Accepted 20 April 2005
$\mathrm{T}$ he congenital coronary artery anomalies that present in adulthood are relatively uncommon. However, ectopic origin of a coronary artery from the aorta is a diagnosis that must be excluded in young adults with typically ischaemic sounding chest pain or syncope. These anomalies have a high clinical profile and are the second most common cause of sudden exertion related cardiac death in athletes. ${ }^{12}$ Most cardiologists will see many such patients during their career and should be prepared to investigate them appropriately, which includes ruling out an anomalous coronary artery as the cause of the symptoms. More commonly these anomalies are found incidentally at coronary angiography. Not infrequently the three dimensional course of the coronary artery with an ectopic origin is incompletely defined under these circumstances. Other rare anomalies that present in adulthood, such as coronary artery fistulae, are potentially more challenging to delineate by means of cardiac catheterisation. ${ }^{3}$ Full, non-invasive assessment of congenital coronary artery anomalies or their exclusion may therefore be advantageous in various circumstances. Echocardiography may provide a definitive diagnosis but traditionally magnetic resonance (MR) imaging has been the noninvasive imaging modality of choice for congenital coronary artery anomalies. ${ }^{4}$

Multidetector row computed tomography (MDCT) has rapidly evolved from the research setting to become a useful clinical tool and must now also be considered for this role. Submillimetre, ECG gated MDCT allows rapid acquisition of a whole heart, three dimensional volume of data in one scan. Importantly it has superior spatial resolution to MR imaging and as a result it appears to be a promising tool for noninvasive diagnostic coronary angiography. ${ }^{5-10}$ The quality of the imaging of the main coronary arteries and side branches provided by MDCT (fig lA) may have importance when assessing congenital coronary artery anomalies. We review the rationale for using MDCT for this indication and examine the advantages and disadvantages of the technique. We present examples of MDCT imaging of congenital coronary artery anomalies, which provide persuasive evidence to support the use of this imaging tool.

\section{SCOPE AND NATURE OF THE PROBLEM}

Congenital coronary artery anomalies affect about $1 \%$ of the population with $87 \%$ of these having anomalies of origin and distribution and $13 \%$ having coronary artery fistulae. ${ }^{11-13}$ The true incidence of ectopic origin of the coronary arteries from the aorta in the population is unknown, with estimates in other series of 0.17$0.6 \% .^{14}{ }^{15}$ Coronary artery fistulae themselves account for $<1 \%$ of all congenital heart defects. ${ }^{16}$ Males and females appear to be equally affected and no hereditary or epidemiological predilection has been observed. These anomalies are rarely recognised in infancy and are increasingly common in adolescents and young adults.

Several different classifications for congenital coronary artery anomalies have been suggested. ${ }^{17-19}$ The most comprehensive of these classifications is that proposed by Angelini et al. ${ }^{20}$ Their favoured definitions are as follows: normal, any morphological feature observed in $>1 \%$ of an unselected population (fig 1B); normal variant, an alternative, relatively unusual morphological feature seen in $>1 \%$ of the same population (fig 2); and anomaly, a morphological feature seen in $<1 \%$ of that population.

Anomalies may then be classified into those of origin and course, intrinsic coronary arterial anatomy, and coronary termination.

\section{ORIGIN AND COURSE}

Anomalous origins are varied and well documented. An anomalous coronary artery origin is considered when the ostium is located above the sinotubular junction (fig 3), in the posterior aortic sinus, or close to the aortic commissure. Left and right coronary arteries may also arise from their opposite coronary sinuses (fig 4), the

Abbreviations: $C T$, computed tomography; MDCT, multidetector row computed tomography; MR, magnetic resonance 

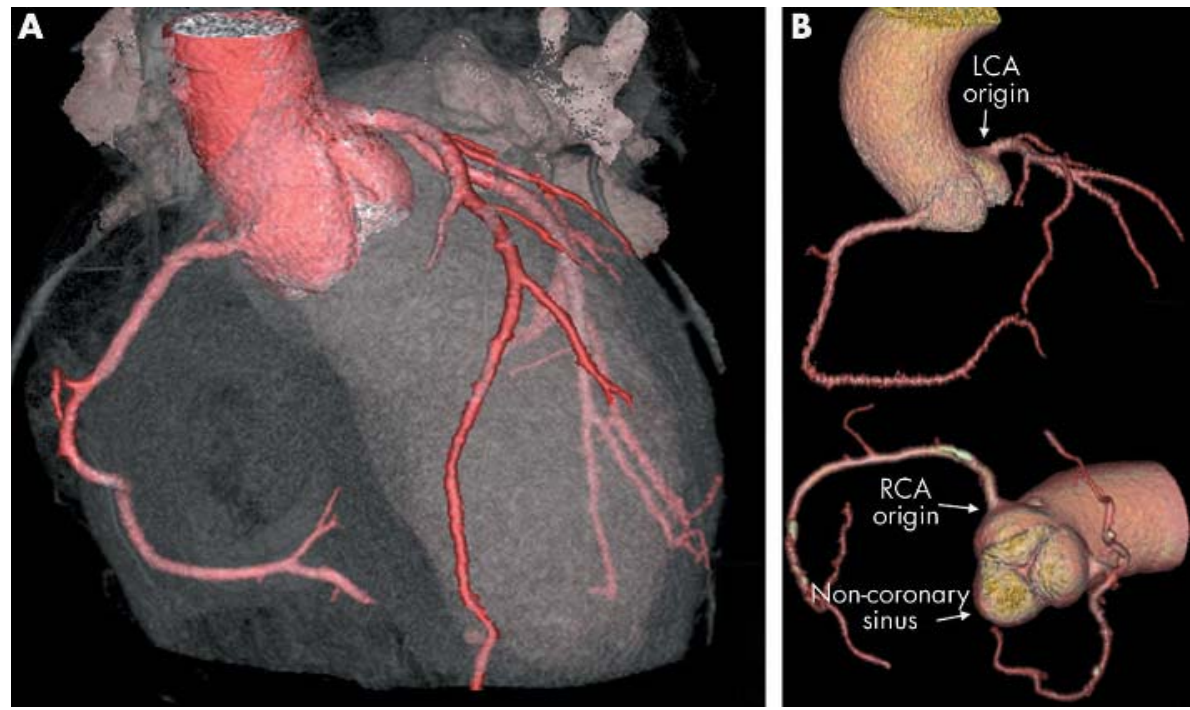

Figure 1 (A) Cardiac transparency and (B) three dimensional contrast vessel tracking tree volume rendering (VR) (GE Medical Systems) protocols show a normal aortic root and coronary artery anatomy. Note the calcification in the right coronary artery (RCA). LCA, left coronary artery.

non-coronary sinus, or indeed from the opposite artery, which may involve joint origination or adjacent double ostia (fig 5). The functional significance of these arrangements, however, more importantly depends on the course taken by the artery rather than the anomalous origin. These courses include (a) passage anterior to the right ventricular outflow tract (fig 6); (b) passage posterior to the aorta; (c) passage between the aorta and right ventricular outflow tract (fig 7, fig 8), where it poses a potential risk that has been convincingly established; and (d) myocardial bridging or intramyocardial tunnelling (fig 9). ${ }^{21-24}$ Patients with clinically significant anomalies generally present before midlife and often in the context of strenuous physical exercise. ${ }^{25}$ Functional compromise is usually the result of hypoperfusion and resultant myocardial ischaemia leading to angina, which

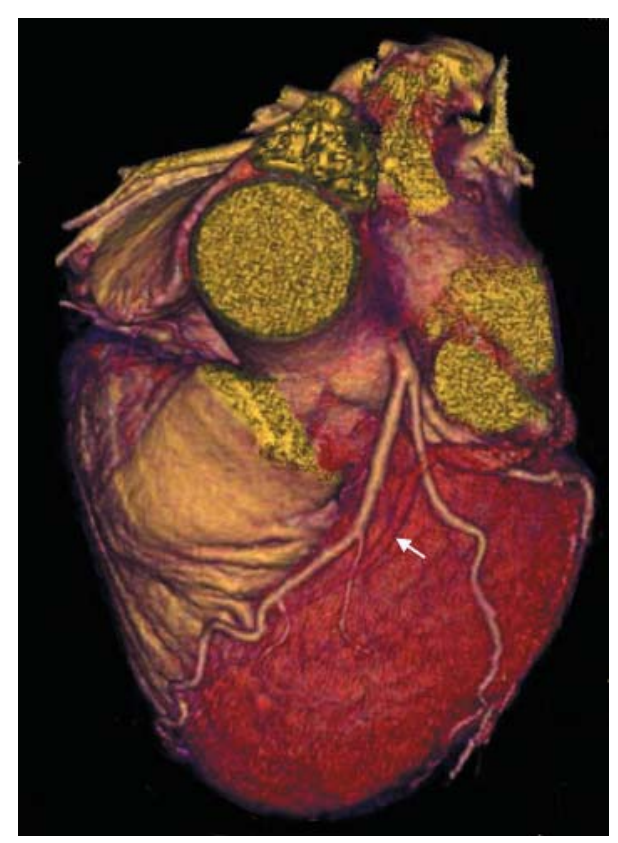

Figure 2 Three dimensional VR image showing normal variant trifurcation anatomy of the left main stem artery. The left atrial appendage and the pulmonary oufflow tract have been removed to reveal the LCA origin. Note also the great cardiac vein passing over the proximal branches (arrow). itself may present typically or atypically. Cardiac arrhythmias, particularly those of ventricular origin, or syncope may also be presenting features. Coronary artery bypass surgery or coronary reimplantation is considered under theses circumstances.

Anomalous origin may also be the ventricles, pulmonary artery, and aortic arch, with other extracardiac origins such as the descending aorta and innominate, subclavian, carotid, and internal mammary or bronchial arteries; these are, however, extremely rare. Anomalous origin of the left coronary artery from the pulmonary trunk is the most common major congenital malformation of the coronary circulation (about 1:300 000 live births), but this manifests itself in infancy.

\section{INTRINSIC CORONARY ARTERIAL ANATOMY}

The presence of several anomalies of intrinsic coronary arterial anatomy such as ostial stenosis, atresia, and single, absent, or hypoplastic coronary arteries may have clinical importance. These anomalies may be indicated as a cause of sudden cardiac death and reconstructive surgery may be considered.

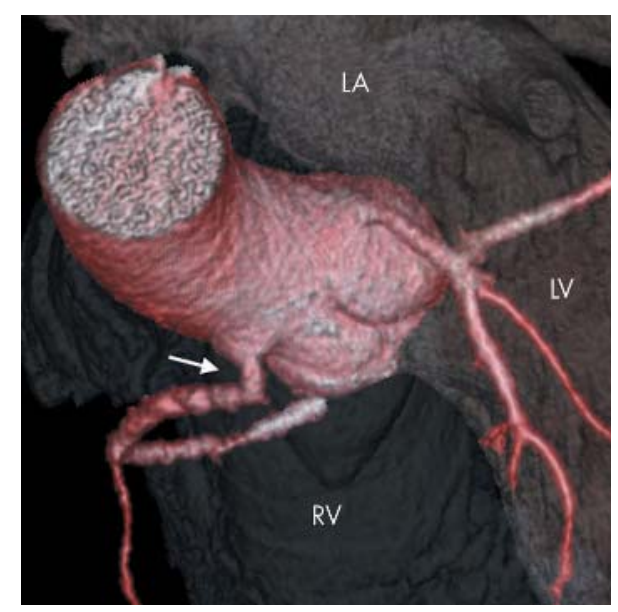

Figure 3 Both the LCA and RCA arise from the ascending aorta above the aortic sinuses. Note also the sharp angulation of the proximal RCA (arrow). LA, left atrium; LV, left ventricle; RV, right ventricle. 

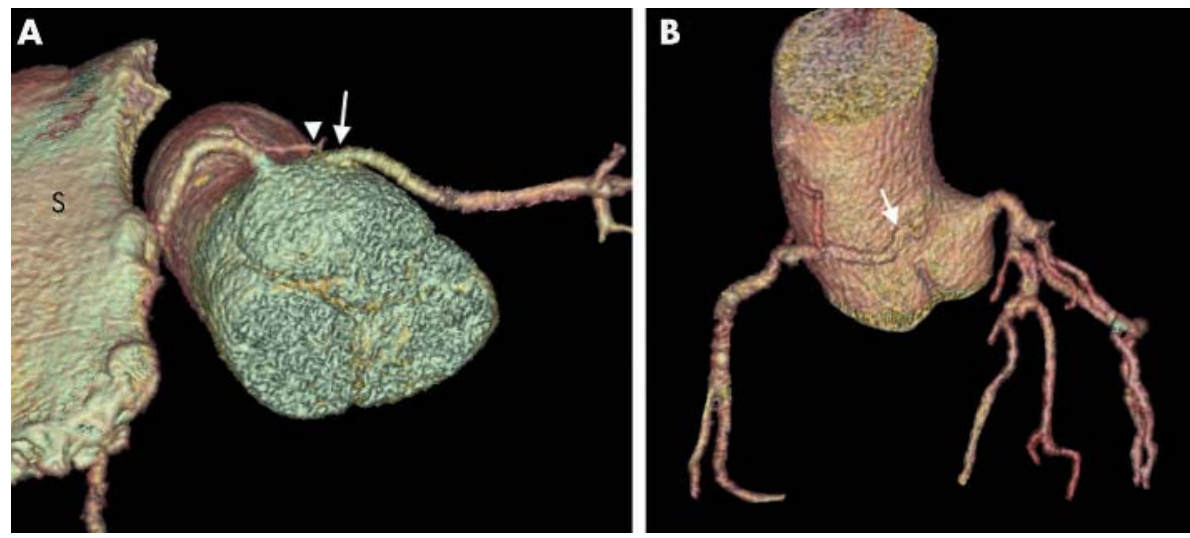

Figure 4 (A) This patient with Marfan's syndrome and notable pectus excavatum (note the sternum $(S)$ with the RCA just posterior to it) has an anomalous LCA arising from the right aortic sinus (arrow). Note also the conus artery arising from this sinus (arrowhead). (B) The RCA arises above the opposite aortic sinus (arrow).

\section{CORONARY TERMINATION: CORONARY ARTERY FISTULAE}

Both the left and right coronary arteries arise normally from the aorta. Commonly the right coronary artery is affected, although left sided coronary artery fistulae are well documented (fig 10). ${ }^{26}$ Previously fistulae have been reported as usually draining into the pulmonary artery or right ventricle, but the fistulae may drain to anywhere from between the vena cava or coronary sinus to the pulmonary artery or left atrium. $^{3}$ Multiple fistulae may also occur. Over $90 \%$ of fistulae drain into the right side of the heart and therefore cause a shunt. Chronic large volume shunts through these fistulae may cause major aneurysmal enlargement of the proximal feeding coronary artery (fig 11) in addition to enlargement of the receiving vessel or chamber (fig 12A). The uninvolved coronary artery and chambers are usually normal.

Coronary artery fistulae may cause symptoms as a result of right heart dilatation (fig 12B) and pressure overload or as a result of coronary steal, leading to breathlessness, chest pain, or arrhythmias. The symptoms depend on the site of fistula entry, the volume and duration of flow, and the presence of significant obstruction to flow at that entry site. Presentation may also be with fistula rupture, tamponade, or bacterial endocarditis. Once the patient is symptomatic there is general consensus that the fistula should be closed and this may be accomplished surgically or by using catheter based techniques. The largest reported experience of transcatheter closure ( 33 patients over a 13 year period) of coronary artery fistulae describes a predominantly paediatric population. ${ }^{3}$ A larger experience of surgical closure is reported in the literature with more emphasis on adult patients. ${ }^{27}$ Aneurysm formation (fig 13A) and vessel tortuosity (fig 13B) both increase with age. These factors tend to make surgical treatment more appropriate but in general the mode of fistula closure depends greatly on local expertise. ${ }^{3}$

\section{AVAILABLE IMAGING MODALITIES}

Cardiac catheterisation has traditionally been indicated to detail the coronary vasculature before intervention and has remained the reference standard imaging modality. Recognition of important clues and specific angiographic views are required to delineate fully many coronary arterial anomalies. Owing to the potentially complex three dimensional nature of these anomalies, conventional angiography, not infrequently, incompletely delineates the anatomical course of the coronary artery. Invasive coronary angiography for fistulous anomalies requires a catheter in the right ventricular outflow tract and multiple views to define. Distal occlusion devices and power injection are often needed for coronary artery flow evaluation to define side branches and aneurysms for complete assessment. ${ }^{3}$ Reliable, complete, non-invasive assessment (or indeed reliable exclusion of congenital coronary artery anomalies) is therefore desirable and advantageous.

Transthoracic echocardiography is a practical and often diagnostic test if specific attention is paid to the coronary arteries. Most patients with suspect symptoms should undergo transthoracic echocardiography. This may provide a diagnosis by identifying anomalous arterial origins, delineate entire fistulous courses, and characterise their haemodynamic status and the size and function of receiving chambers, which may reflect potentially adverse effects of a coronary steal. The accuracy of echocardiography is operator dependent and limited by patient anatomy and optimal
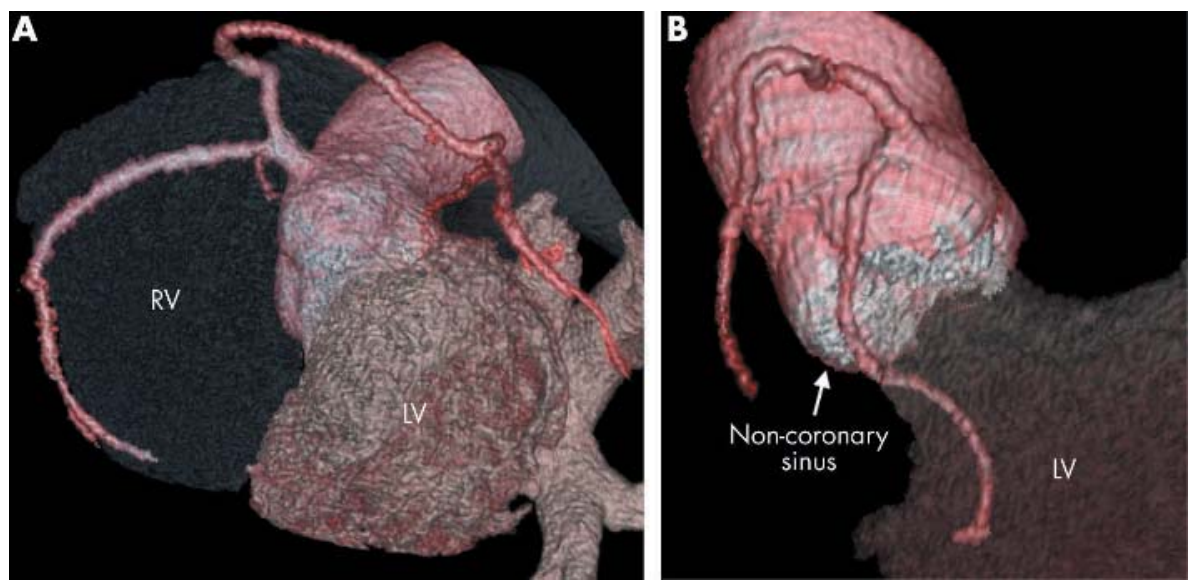

Figure 5 (A) The LCA arises from a common origin with the RCA from the right aortic sinus. (B) The circumflex artery arises separately close to the origin of the RCA and passes below the non-coronary sinus. 


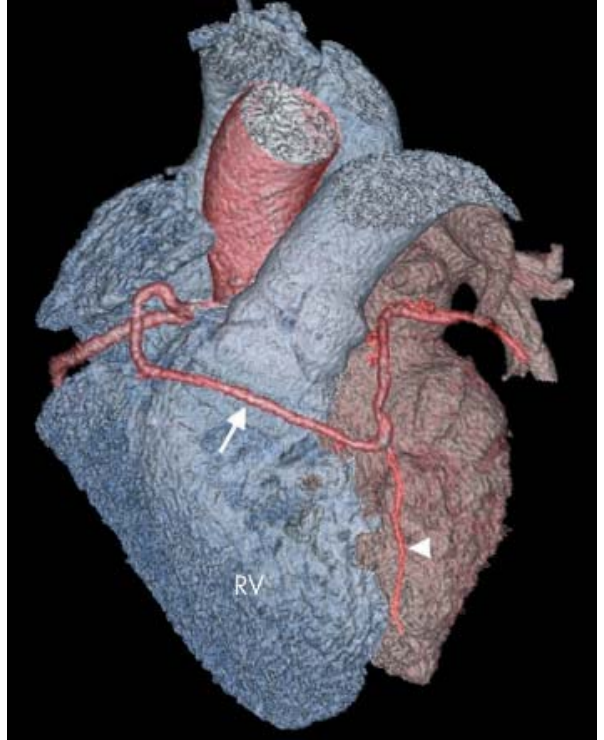

Figure 6 Anomalous LCA arising from a common RCA origin and coursing anterior to the RVOT (arrow). Note the vestigial left anterior descending artery (LAD) (arrowhead).

acoustic windows. Even transoesophageal echocardiography may fail to completely delineate coronary anomalies, and it is not totally non-invasive.

Other non-invasive techniques such as nuclear and cineMR cardiac imaging may become increasingly important. Traditionally MR imaging has been the non-invasive imaging modality of choice for the identification of congenital coronary artery anomalies and their courses. ${ }^{428} \mathrm{New}$ MR imaging sequences have improved image quality with better anatomical definition and have become an alternative means to evaluate flow and function. ${ }^{29}$

The spatial resolution achievable with MR imaging is marginal for coronary artery imaging, however, and its greatest limitation is in determining the distal arterial course. $^{30}$ In comparison, MDCT cardiac imaging provides excellent distal coronary artery and side branch visualisation. Furthermore, if required, an entire three dimensional volume of the heart and great vessels may be acquired within 20 seconds.
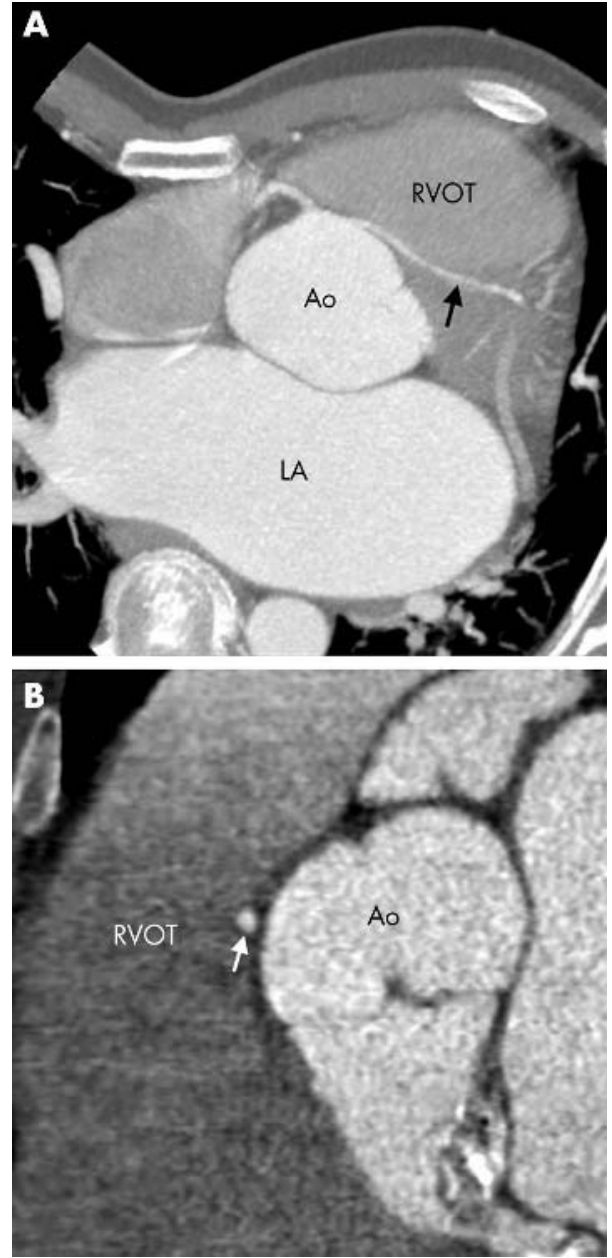

Figure 8 (A) Axial image of the same patient as in fig 4A also shows the malignant course taken by the anomalous LCA coursing between the RVOT and Ao (arrow). (B) Sagittal (parasternal) image.

\section{RATIONALE FOR MDCT IMAGING Spatial and temporal resolution}

Four slice computed tomography (CT) has been extensively evaluated for coronary artery imaging; however, CT coronary angiography with this level of technology was not sufficiently
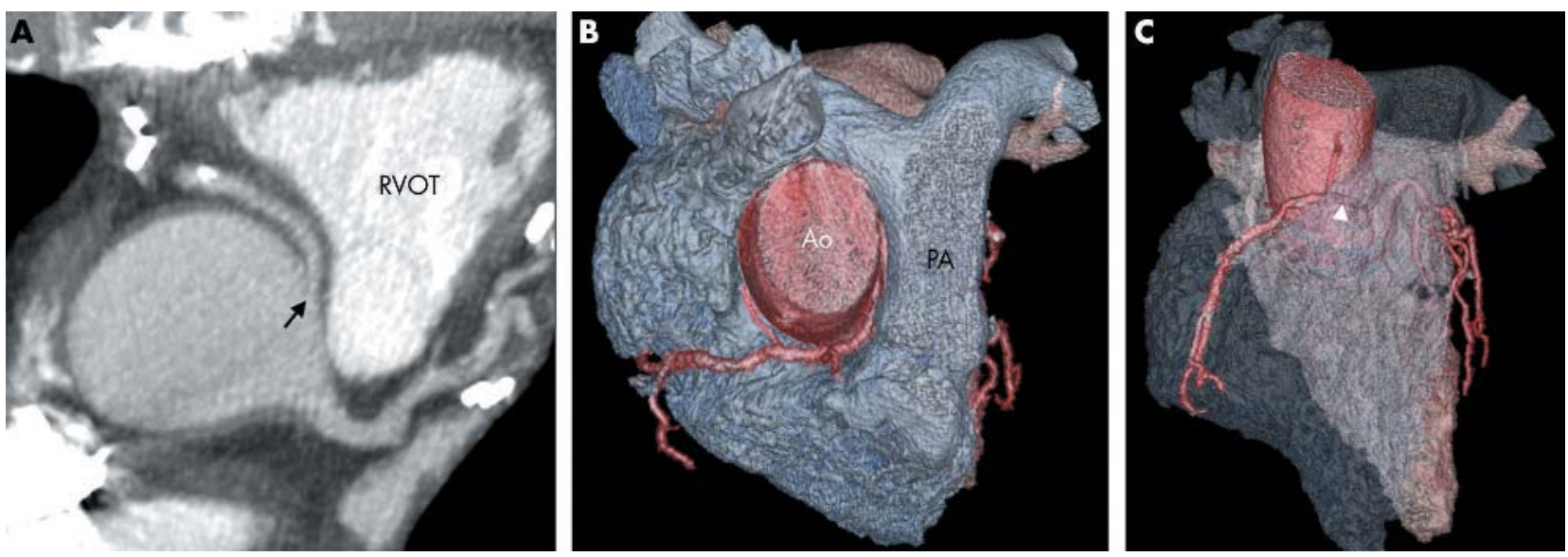

Figure 7 (A) Axial thick slab maximum intensity projection (MIP) image shows an anomalous RCA originating from the left coronary sinus (arrow) and coursing between the aorta and RVOT. (B, C) Cardiac transparency assists in visualising this potentially malignant course (arrowhead). Ao, aorta; PA, pulmonary artery. 

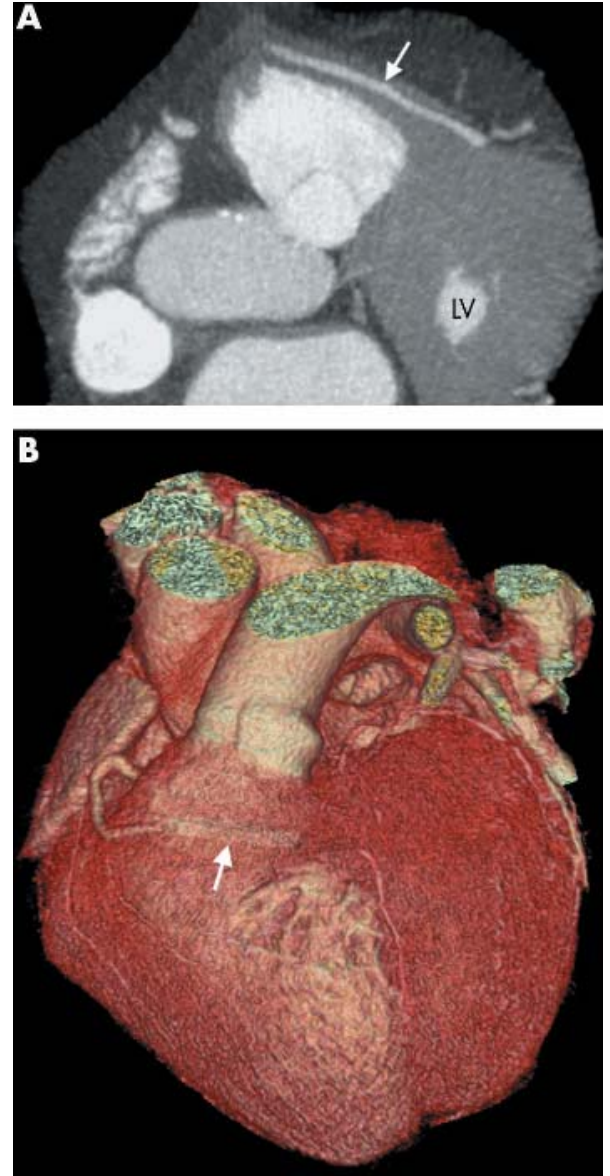

Figure 9 (A) Axial thick MIP and (B) three dimensional VR images show an anomalous LCA passing anterior to the RVOT while taking a prolonged intramyocardial course (arrow) where the vessel may become compressed.

robust to produce consistent and reliable imaging for clinical use in terms of both reliability of image quality and accuracy of results. ${ }^{31-35}$ Specifically, high heart rates induce motion artefact, and excessive coronary artery calcification impedes accurate lumen visualisation by producing "partial voluming averaging" artefacts leading to an overestimation of the degree of stenosis and thus a false positive detection. ${ }^{36}$

Sixteen slice CT has recently superseded four slice CT. For cardiac applications this technology provides not only a submillimetre collimation but also an improved temporal resolution. Importantly it has a superior spatial resolution to MR imaging and as a result the technique has reached an acceptable level of diagnostic accuracy for non-invasive diagnostic coronary angiography. ${ }^{5-10} \mathrm{CT}$ coronary angiography now appears to be clinically useful. In addition, evaluation of adult congenital heart disease with ECG gated MDCT imaging has increasingly been found to be a useful tool. ${ }^{37}$ Electron beam CT has also been used to visualise the anatomical course of congenitally abnormal coronary arteries $^{38}$ and, more recently, MDCT has also been shown to characterise the proximal courses in nine patients. ${ }^{39}$

\section{ECG gating}

Cardiac motion artefact caused by pulsation and rotational movement may be minimised, with concurrent high spatial resolution, if the scanned volume of data is reconstructed at time points when cardiac motion is least: this occurs during diastole. Heart phase data are acquired with reference to the simultaneously acquired ECG trace. MDCT is prospectively
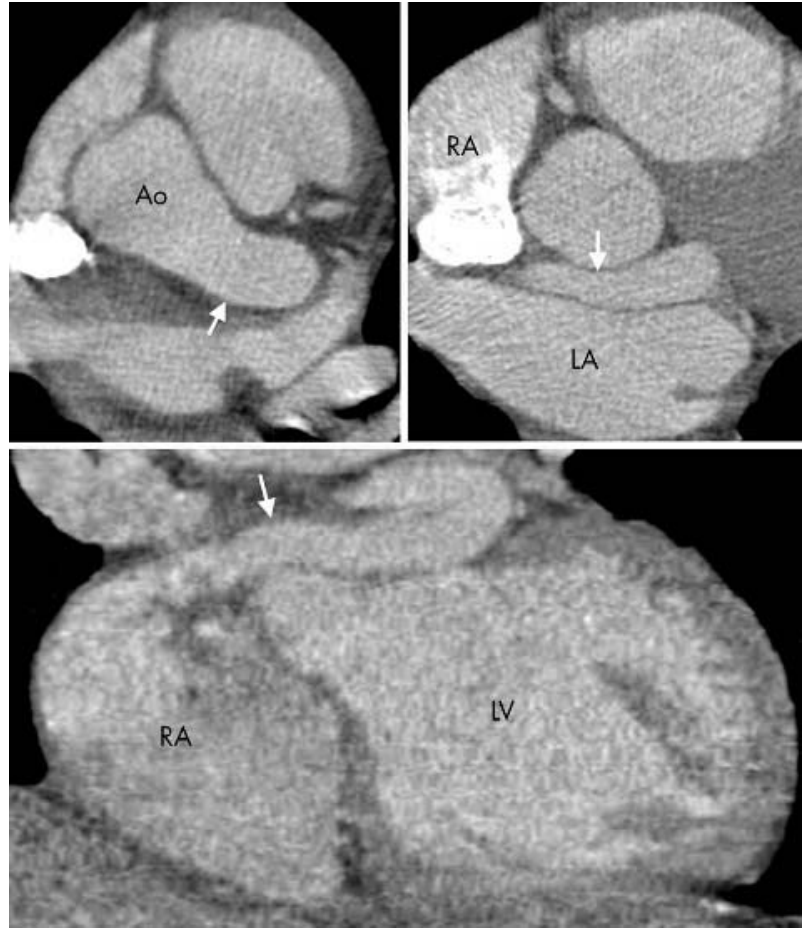

Figure 10 Axial section at the level of the left main stem (upper left), a further more inferior section (upper right), and a coronal section (lower) show an LCA fistula (arrows), which passes behind the left aortic sinus, anterior to the LA, and enters the right atrium (RA). The vessel, which is aneurysmal from the point of the left main stem origin, gave off the LAD and circumflex artery.

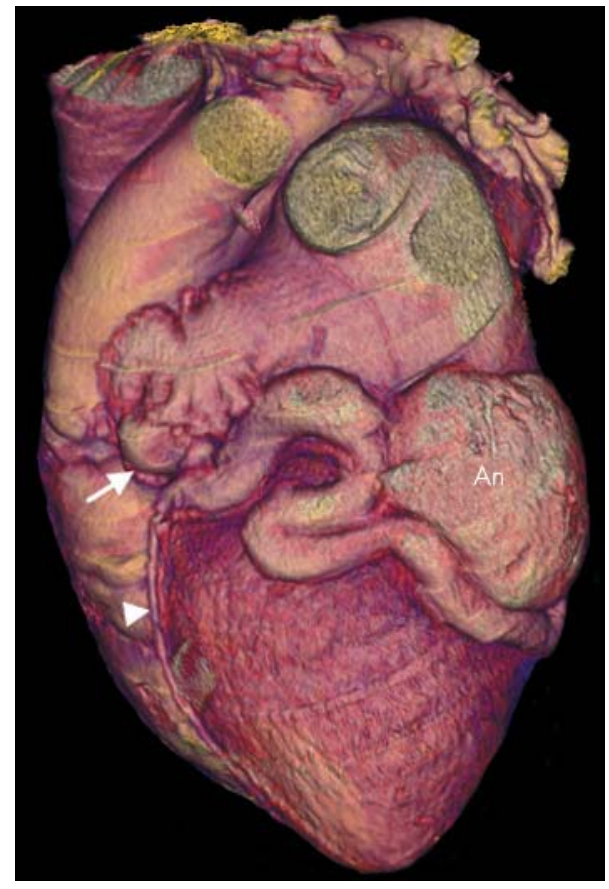

Figure 11 Three dimensional VR image showing a circumflex artery fistula. The left main stem is greatly dilated (arrow) and a dilated, tortuous circumflex artery becomes aneurysmal (An) before draining into the coronary sinus. Note also the LAD arising from this dilated vessel (arrowhead). 

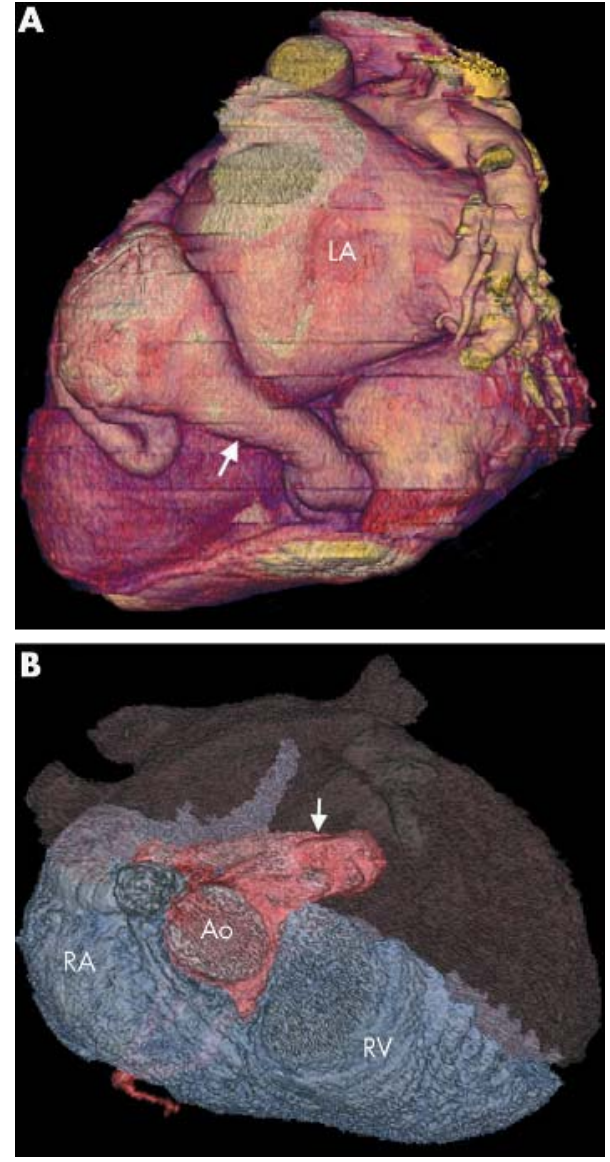

Figure 12 (A) Three dimensional VR (posterior view) of the circumflex artery fistula showing major dilatation of the receiving vessel (the coronary sinus; arrow). (B) This VR superior view helps to show the LCA fistula (arrow) in the RA and subsequent dilatation of the right heart chambers.

triggered, or gated, during sequential imaging, and retrospectively gated during continuous spiral imaging (fig 14). Essentially, during prospective gating, the scan is triggered at a defined position along the $\mathrm{R}$ wave to $\mathrm{R}$ wave interval during consecutive heart beats, usually during late diastole. The examination time is thus related to overall heart rate. Prospective imaging is heavily dependent on a regular heart rate with artefact and misregistration of data occurring in the presence of cardiac arrhythmias.

Retrospective gating allows for greater flexibility. This method acquires phase data continuously throughout the cardiac cycle and necessitates multidetector scanning allowing images to be reconstructed and viewed at any point along the $\mathrm{R}-\mathrm{R}$ interval. ${ }^{40}$ Retrospective ECG gating is thus the method of choice for contrast enhanced images of the coronary arteries in high spatial resolution.

Imaging in late diastole or about $75 \%$ of the $\mathrm{R}-\mathrm{R}$ interval is the phase of least cardiac motion for the majority of coronary artery segments. Individual patients may have portions of cardiac anatomy with motion patterns that do not conform to this, requiring analysis on different phases ${ }^{41}$ The duration of the diastolic phase with minimal cardiac motion is directly related to the heart rate. Most studies suggest that heart rate is inversely proportional to image quality on CT coronary angiography. ${ }^{42}{ }^{43}$ At higher heart rates, good quality imaging is achievable but this is less reproducible, so slower heart rates are preferred in order to achieve consistently high quality coronary arterial imaging.
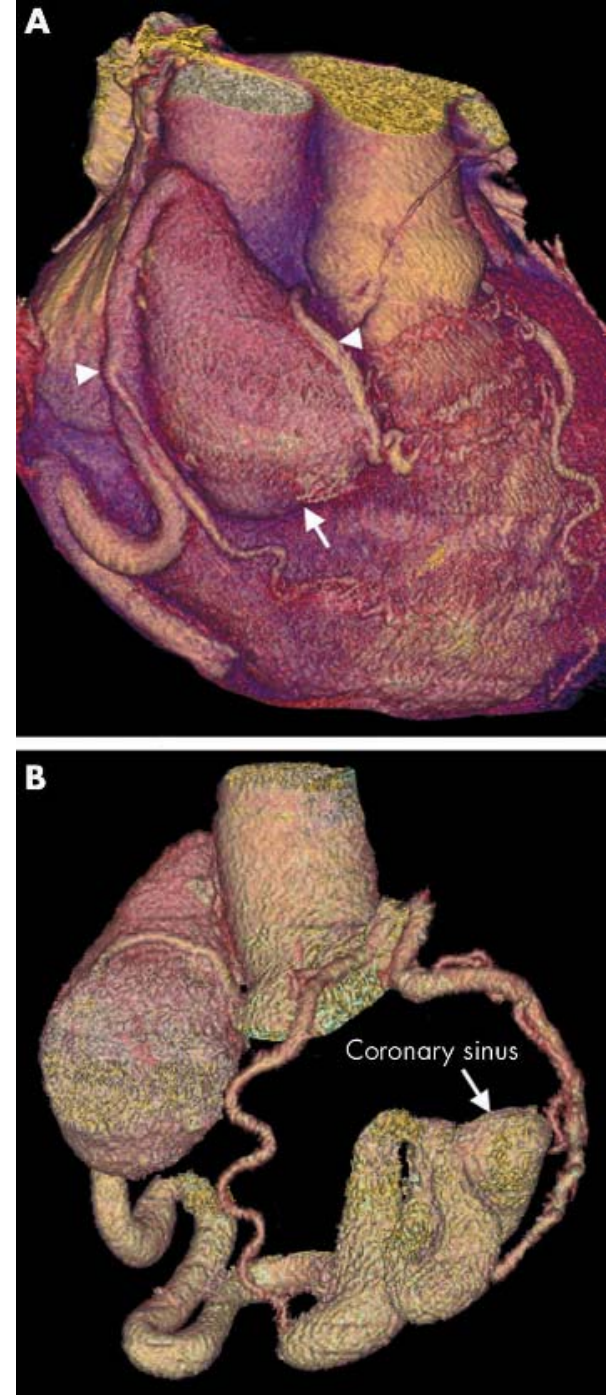

Figure 13 (A) Three dimensional VR image showing a large RCA aneurysm (arrow). The RCA then continues as a dilated tortuous vessel. Two RCA branches are clearly shown passing down on to the RV (arrowheads); these were not visible on conventional angiography. (B) The aneurysm has formed secondary to an RCA fistula draining into the coronary sinus and then the RA. Its tortuous, dilated course can also be fully delineated by three dimensional vessel tracking techniques. Note the normal left circulation.

Submillimetre, ECG gated MDCT allows rapid acquisition of an entire three dimensional cardiac volume of data in a single, rapid scan.

Even higher isotropic spatial resolution is possible with the next generation of CT technology, the 32 detector and 64 channel CT. The 64 channel detectors are being developed with recent results indicating the visual clarity of up to fifth order coronary arterial branches. ${ }^{44}$ The clinical impact of this new CT technology, particularly for cardiac applications, remains to be studied, though it seems to be the future of CT coronary angiography.

\section{Post-processing}

Given the inherent tortuosity and variability of coronary arterial anatomy and particularly anomalies, evaluation of CT axial data alone is often insufficient. Semiautomated, interactive post-processing display protocols may be used to facilitate a more accurate appreciation of the entire complex 
R-R interval

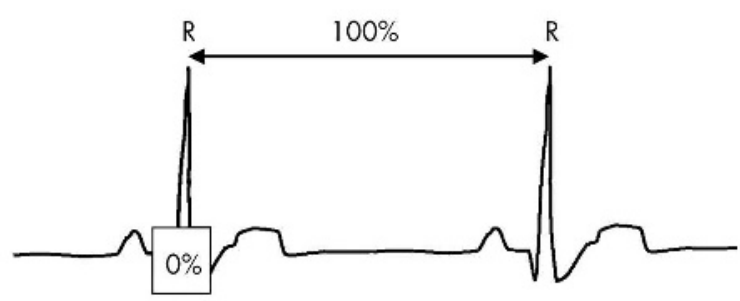

Prospective gating

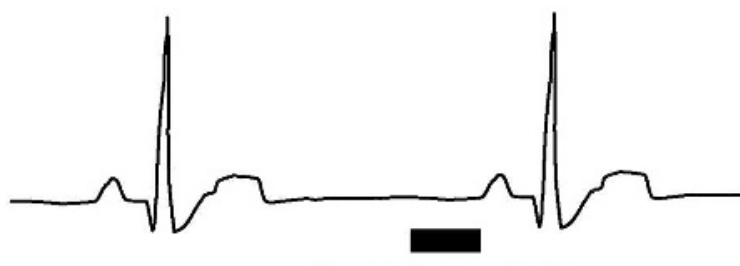

Scan and reconstruction

Retrospective gating

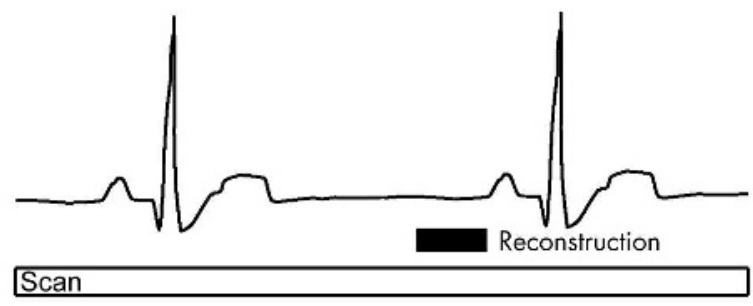

Figure 14 During prospective gating, the scan is triggered at a defined position along the R-R interval during consecutive heart beats. Retrospective gating acquires phase data continuously throughout the cardiac cycle, allowing images to be reconstructed and viewed at any point along the $R-R$ interval.

three dimensional anatomy or simply to display certain regions of interest.

Post-processing software in three dimensions, commonly by three dimensional volume rendering or through cardiac cine or movie loops, can make this complex data display more intuitive and sometimes quite dramatic. These methods have been augmented by the use of other more experimental algorithms whereby entire arterial courses can be "grown" by tracking a contrast enhanced vessel.

The resulting images provide clinicians with more understandable results and facilitate accurate surgical planning.

\section{Radiation}

It must be remembered that MDCT scanners can achieve higher tube currents with improved image quality but with an increased patient radiation burden. Radiation exposures are relatively high in MDCT coronary angiography ranging from $5-10 \mathrm{mSv} .{ }^{45}$ However, to put the patient radiation dose into context, if the investigation proposed supplies predictable, accurate diagnostic information about the coronary arteries, the effective radiation doses (with ECG controlled tube current modulation) are quite acceptable for clinical use. Indeed, under these circumstances, the dose compares favourably with that of invasive coronary angiography (5$7 \mathrm{mSv}) .{ }^{46}$

\section{CONCLUSION}

The improvements in spatial and temporal resolution, sophisticated ECG gating, and post-processing software algorithms are allowing motion-free, fast, accurate, and detailed contrast enhanced cardiac imaging that rivals the accuracy of traditional invasive and non-invasive diagnostic techniques.

We must determine, however, where exactly MDCT should be used for optimum clinical and cost effective benefit.

The identification and evaluation of congenital anomalies of the coronary arteries seem to be appropriate clinical uses for this technology and the examples illustrated in this review provide persuasive evidence in support of this clinical use. Long term observation and follow up of these patients may also be an appropriate use of CT to clarify the particular anomaly's natural progression.

A clinical group of patients who may acquire particular benefit from non-invasive imaging with CT are young patients who have exertion related ischaemic pain, syncope, or ventricular tachyarrhythmias and who would not be expected to have significant underlying calcific atherosclerosis, which may otherwise compromise this technique's sensitivity.

The authors thus advocate consideration of the clinical use of MDCT cardiac imaging in the context of suspected congenital coronary artery anomalies as a first line investigation.

\section{ACKNOWLEDGEMENTS}

Dr Nathan Manghat is funded by The Royal College of Radiologists Research Fellowship Award 2004/5 to study the clinical applications of cardiac computed tomography.

The authors thank all the Derriford Hospital CT radiographers for all their efforts in supporting our cardiac clinical work and research.

\section{Authors' affiliations}

N E Manghat, C A Roobottom, Department of Clinical Radiology, Plymouth NHS Trust, Derriford, Plymouth, UK

G J Morgan-Hughes, A J Marshall, South West Cardiothoracic Centre, Plymouth NHS Trust, Derriford, Plymouth, UK

The authors declare no competing interests

\section{REFERENCES}

1 Liberthson R. Sudden cardiac death in the child and young adult. N Engl J Med 1996;334:1035-44.

2 Corrado D, Basso C, Rizzoli G, et al. Does sports activity enhance the risk of sudden death in adolescents and young adults? J Am Coll Cardiol 2003;42:1959-63.

3 Armsby L, Keane J, Sherwood M, et al. Management of coronary artery fistulae patient selection and results of transcatheter closure. J Am Coll Cardiol 2002;39:1026-32.

4 Parga J, Ikari N, Bustamante N, et al. MRI evaluation of congenital coronary artery fistulae. Br J Radiol 2004;77:508-11.

5 Nieman K, Cademartiri F, Lemos P, et al. Reliable non-invasive coronary angiography with fast submillimeter multislice spiral computed tomography. Circulation 2002;106:2051-4.

6 Ropers D, Baum U, Pohle K, et al. Detection of coronary artery stenosis with thin-slice multi-detector row spiral computed tomography and multiplanar reconstruction. Circulation 2003; 107:664-6.

7 Heuschmid M, Kuttner A, Flohr T, et al. [Visualisation of coronary arteries in $C T$ as assessed by a new 16 slice technology and reduced gantry rotation time: first experiences/. Rofo 2002;174:721-4.

8 Morgan-Hughes GJ, Roobottom CA, Owens PE, et al. Highly accurate coronary angiography with submillimetre, 16 slice computed tomography. Heart 2005;91:308-13.

9 Mollet N, Cademartiri F, Nieman K, et al. Multislice spiral computed tomography coronary angiography in patients with stable angina pectoris. J Am Coll Cardiol 2004:43:2265-70.

10 Kuettner A, Trabold T, Schroeder S, et al. Noninvasive detection of coronary lesions using 16-detector multislice spiral computed tomography technology: initial clinical results. J Am Coll Cardiol 2004;44:1230-7.

11 Baltaxe $\mathrm{H}$, Wixson D. The incidence of congenital anomalies of the coronary arteries in the adult population. Radiology 1977;122:47-52.

12 Click RL, Holmes DR Jr, Vlietstra RE, et al. Anomalous coronary arteries: location, degree of atherosclerosis and effect on survival: a report from the coronary artery surgery study. J Am Coll Cardiol 1989;3:531-7. 
13 Yamanaka O, Hobbs R. Coronary artery anomalies in 126,595 patients undergoing coronary angiography. Cathet Cardiovasc Diagn 1990;21:28-40.

14 Liberthson RR, Dinsmore RE, Fallon JT. Aberrent coronary artery origin from the aorta: report of 18 patients, review of the literature, and delineation of natural history and management. Circulation 1979;59:748-54.

15 Davis JA, Cecchin F, Jones TK, et al. Major coronary artery anomalies in a paediatric population: incidence and clinical importance. J Am Coll Cardiol 2001;37:593-7.

16 Lithbertson R, Sagar K, Berkoben J, et al. Congenital coronary artery fistula: report of 13 patients, review of the literature and delineation of management. Circulation 1979;59:849-54

17 Blake HA, Manion WC, Mattingley TW, et al. Coronary artery anomalies. Circulation 1964:30:927-34.

18 Ogden JA. Congenital anomalies of the coronary arteries. Am J Cardiol 1970;25:474-9.

19 Roberts WC. Major anomalies of coronary arterial origins seen in adulthood. Am Heart J 1986; 111:941-63.

20 Angelini P, Velasco J, Flamm S. Coronary anomalies: incidence, pathophysiology, and clinical relevance. Circulation 2002;105:2449-54.

21 Kragel AH, Roberts WC. Anomalous origin of either the right or left main coronary artery from the aorta with subsequent coursing between aorta and pulmonary trunk: analysis of 32 necropsy cases. Am J Cardiol 1988:62:771-7.

22 Taylor AJ, Rogan KM, Virmani R. Sudden cardiac death associated with isolated congenital coronary artery anomalies. J Am Coll Cardiol 1992;20:640-7.

23 Thomas D, Salloum J, Montalescot G, et al. Anomalous coronary arteries coursing between the aorta and pulmonary trunk: clinical indications for coronary artery bypass. Eur Heart J 1991;12:832-4.

24 Morales AR, Romanelli R, Tate LG, et al. Intramural left anterior descending coronary artery: significance of the depth of muscular tunnels. Hum Pathol 1993;24:693-701.

25 Eckart RE, Scoville SL, Campbell CL, et al. Sudden death in young adults: a 25 year review of autopsies in military recruits. Ann Intern Med 2004;141:829-34.

26 Gandy K, Rebeiz A, Wang A, et al. Left main coronary artery to pulmonary artery fistula with severe aneurysmal dilatation. Ann Thorac Surg 2004;77:1081-3.

27 Goto $Y$, Abe T, Sekine S, et al. Surgical treatment of the coronary artery to pulmonary artery fistulas in adults. Cardiology 1998;89:252-6.

28 McConnell MV, Ganz P, Selwyn AP, et al. Identification of anomalous coronary arteries and their anatomic course by magnetic resonance coronary angiography. Circulation 1995;92:3158-62.

29 Duerinckx AJ, Shaaban A, Lewis A, et al. 3D MR imaging of coronary arteriovenous fistulas. Eur Radiol 2000;10:1459-63.
30 Wielopolski PA, van Geuns RJM, de Feyter PJ, et al. Coronary arteries. Eur Radiol 1998;8:873-85.

31 Achenbach S, Ulzheimer S, Baum U, et al. Noninvasive coronary angiography by retrospectively ECG-gated multislice spiral CT. Circulation 2000;102:2823-8.

32 Nieman K, Oudkerk $M$, Rensing $B$, et al. Coronary angiography with multislice computed tomography. Lancet 2001;357:599-603.

33 Knez A, Becker C, Leber A, et al. Usefulness of multislice spiral computed tomography angiography for determination of coronary artery stenoses. Am J Cardiol 2001;88:1191-4.

34 Morgan-Hughes G, Marshall A, Roobottom C. Multislice computed tomographic coronary angiography: experience in a UK centre. Clin Radiol 2002:58:378-83.

35 Kopp A, Schröder S, Küttner A, et al. Non-invasive coronary angiography with high resolution multidetector-row computed tomography: results in 102 patients. Eur Heart J 2002;23:1714-25.

36 Flohr T, Schoepf J, Küttner A, et al. Advances in cardiac imaging with 16section CT systems. Acta Radiol 2003;10:386-401.

37 Morgan-Hughes GJ, Marshall AJ, Roobottom CA. Morphologic assessment of adult patent ductus arteriosus using retrospectively ECG-gated multidetector CT. AJR Am J Roentgenol 2003;181:749-54.

38 Ropers D, Moshage W, et al. Visualization of coronary artery anomalies and their anatomic course by contrast-enhanced electron beam tomography and three-dimensional reconstruction. Am J Cardiol 2001;87:193-7.

39 Deibler AR, Kuzo RS, Vohringer $M$, et al. Imaging of congenital anomalies with multislice computed tomography. Mayo Clin Proc 2004;79:1017-23.

40 Ohnesorge B, Flohr T, Becker C, et al. Cardiac imaging by means of electrocardiographically gated multisection spiral CT: initial experience. Radiology 2000;217:564-71.

41 Kopp AF, Schroeder S, Kuettner A, et al. Coronary arteries: retrospectively ECG-gated multi-detector row CT angiography with selective optimization of the image reconstruction window. Radiology 2001;221:683-8.

42 Nieman K, Rensing B, van Geuns R, et al. Non-invasive coronary angiography with multislice spiral computed tomography: impact of heart rate. Heart 2002;88:470-4.

43 Giesler T, Baum U, Ropers D, et al. Noninvasive visualization of coronary arteries using contrast-enhanced multidetector $C T$ : influence of heart rate on image quality and stenosis detection. AJR Am J Roentgenol 2002;179:91 1-6.

44 Knollman FD, Edic PM, Cesmali E, et al. Coronary artery imaging with flatpanel computed tomography [abstract]. Radiology 2002;225(P):538-9.

45 Morin RL, Gerber TC, McCollough CH. Radiation dose in computed tomography of the heart. Circulation 2003:107:917-22.

46 Jakobs T, Becker C, Ohnesorge B, et al. Multislice helical CT of the heart with retrospective ECG gating: reduction of radiation exposure by ECG-controlled tube current modulation. Eur Radiol 2002;12:1081-6.

\section{IMAGES IN CARDIOLOGY}

\section{Mid cavity hypertrophic cardiomyopathy}

A 62 year old woman presented with recurrent episodes of amarosis fugax and headache. She had a past medical history of well controlled essential hypertension, and had been treated for pulmonary tuberculosis two years previously. A routine transthoracic echocardiogram revealed an abnormal left ventricle, with mid cavity thickening giving the appearance of a smaller chamber adjacent to the main left ventricular chamber. Two dimensional pulse wave Doppler demonstrated dynamic flow acceleration in mid cavity. Cardiac catheterisation revealed normal coronary arteries and confirmed mid cavity hypertrophic cardiomyopathy. An intraventricular gradient of $60 \mathrm{~mm} \mathrm{Hg}$ was demonstrated between the two cavities. A 16 slice multidetector computed tomographic (MDCT) scan was performed following injection of $120 \mathrm{ml}$ of non-iodinated contrast; axial images are shown in panel A. Following acquisition, three dimensional reconstruction was performed offline on a GE workstation to generate a 3D image of the ventricular structure (panel B).
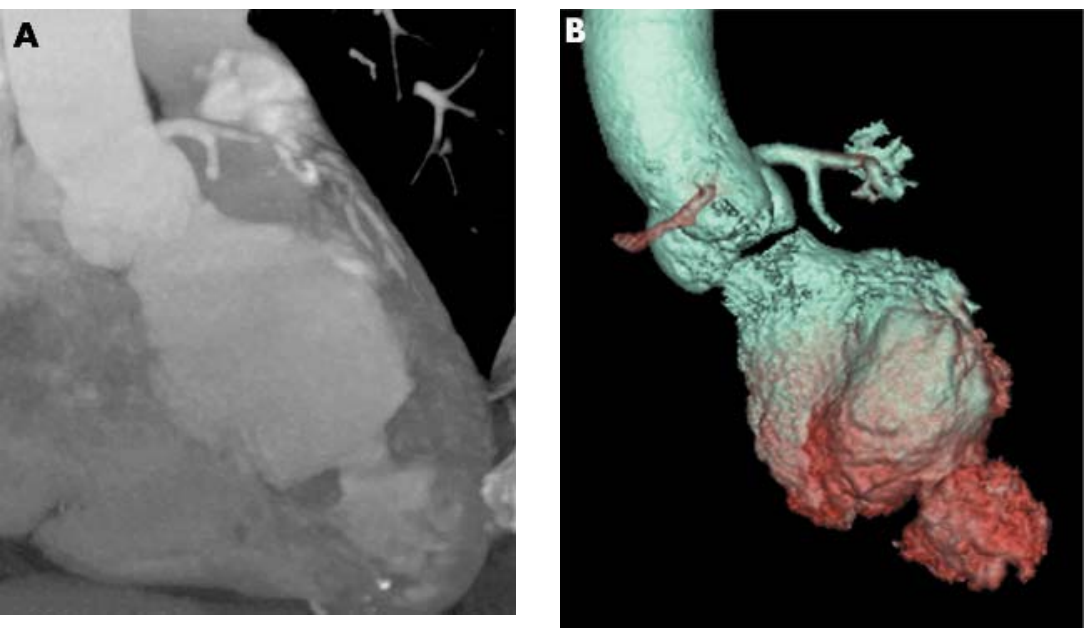